\title{
Prototipo de evaluación genética nacional para fertilidad de vaquillas Simmental-Simbrah y Charolais-Charbray
}

\section{Prototype of national genetic evaluation for heifers fertility in Simmental-Simbrah and Charolais-Charbray}

\author{
Juan José Baeza Rodrígueza ${ }^{a}$ Vicente Eliezer Vega Murillob ${ }^{b}$ Ángel Ríos Utrera ${ }^{b}$, \\ Guillermo Martínez Velázquez ${ }^{c}$, Miguel Enrique Arechavaleta Velascod ${ }^{\mathrm{d}}$, Moisés Montaño Bermúdez ${ }^{\mathrm{d} *}$
}

\begin{abstract}
RESUMEN
El objetivo del presente estudio fue desarrollar un prototipo de evaluación genética multirracial para fertilidad de vaquillas (FERT) en las poblaciones Simmental-Simbrah y Charolais-Charbray. Se utilizó información de 49,943 hembras Simmental-Simbrah, nacidas entre 1984 y 2011 (EvSim) y de 49,871 hembras Charolais-Charbray, nacidas entre 1990 y 2011 (EvChar). Para el análisis, a FERT se le asignó un uno cuando la vaquilla parió antes de los 1,281 días de edad y un cero en caso contrario. Los estimadores de los componentes de varianza y los valores genéticos se obtuvieron ajustando un modelo lineal mixto para una sola característica. Los estimadores de heredabilidad fueron similares, $0.07 \pm 0.008$ y $0.06 \pm 0.008$, para EvSim y EvChar, respectivamente. La mediana del número de hembras por grupo contemporáneo fue 10, en un rango intercuartil de 5 a 20, en ambos casos, EvSim y EvChar. La mediana del número de hembras por familia de medias hermanas paternas fue 5 en ambos casos, EvSim y EvChar. Las diferencias esperadas en la progenie (DEP) de FERT variaron de: -5.79 a 8.72, -9.56 a 8.84, -7.94 a 8.22 , y -7.29 a 6.14 , para Simmental, Simbrah, Charolais y Charbray, respectivamente. Las heredabilidades estimadas indican que puede haber respuesta genética a la selección de FERT en las poblaciones evaluadas. Las tendencias genéticas estimadas muestran un cambio genético favorable en fertilidad de las cuatro razas durante el período 2007-2011. Los rangos de las DEP permiten identificar sementales sobresalientes para FERT.
\end{abstract}

PALABRAS CLAVE: Bovinos carne, Heredabilidad, Tendencias genéticas.

\section{ABSTRACT}

The objective of this study was to develop a prototype of multibreed genetic evaluation for heifers' fertility (FERT) in Simmental-Simbrah and Charolais-Charbray populations. Information from 49,943 Simmental-Simbrah females born between 1984 and 2011 (EvSim) and 49,871 Charolais and Charbray females (EvChar), born between 1990 and 2011 were used. For the analysis, a one was assigned when a heifer calved before 1,281 d of age, otherwise a zero was assigned. Variance components estimates and breeding values were obtained by fitting a linear mixed model for a single trait. Estimates of heritability were similar, $0.07 \pm 0.008$ and $0.06 \pm 0.008$, for EvSim and EvChar, respectively. Median number of heifers per contemporary group was 10, with an interquartile range from 5 to 20, in both cases, EvSim and EvChar. Median number of paternal half sibs per family was $\mathbf{5}$ for both, EvSim and EvChar. The ranges of expected progeny differences (EPD) for FERT were: -5.79 to 8.72, -9.56 to 8.84, -7.94 to 8.22, and -7.29 to 6.14, for Simmental, Simbrah, Charolais and Charbray, respectively. Heritability estimates suggest that there may be genetic response to selection of FERT in the populations evaluated. Estimated genetic trends suggest a favorable genetic change of fertility in the four breeds during the period 2007 to 2011 . The ranges of the EPD allowed identification of outstanding sires for FERT.

KEY WORDS: Beef cattle, Heritability, Genetic trends.

Recibido el 22 de agosto de 2016. Aceptado el 7 de noviembre de 2016.

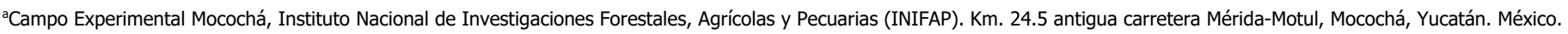

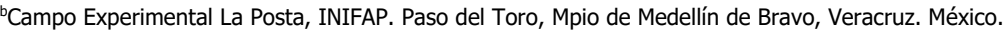

'Campo Experimental Santiago Ixcuintla, INIFAP. Santiago Ixcuintla, Nayarit, México.

${ }^{d}$ CENID - Fisiología, INIFAP. Ajuchitlán Colón, Querétaro. México.

*Autor de correspondencia: montano.moises@inifap.gob.mx. 


\section{INTRODUCCIÓN}

La rentabilidad de los sistemas de producción de carne bovina depende fundamentalmente de la eficiencia reproductiva de la hembra. Se reconoce que las tasas reproductivas altas influyen en forma significativa sobre la eficiencia biológica y económica de los sistemas de producción vaca-cría(1). El impacto económico por mejorar el comportamiento reproductivo puede ser hasta cuatro veces mayor que el logrado por mejorar características de crecimiento y calidad de la canal(2). Los valores de heredabilidad estimados para características reproductivas son bajos, e indican que los efectos ambientales son los principales responsables de la expresión de la fertilidad en vacas y vaquillas ${ }^{(3)}$.

Son pocos los programas nacionales que incluyen evaluaciones genéticas de características reproductivas alrededor del mundo (Estados Unidos, Brasil, Reino Unido y Francia) y pocas las características que se evalúan dentro de cada programa; las principales son preñez de vaquillas, edad al primer parto y permanencia productiva $(3,4)$. Sólo en algunos de estos países las diferencias esperadas en la progenie (DEP), como estimadores del mérito genético de la fertilidad de vaquillas, están disponibles para algunas razas, y se han asociado positivamente con la fertilidad en la fase adulta $^{(4,5)}$.

Resultados recientes muestran la posibilidad de lograr progreso genético en características reproductivas a través de selección. Heredabilidad de $0.49 \pm 0.03$ obtenida para tasa de gestación de vaquillas en ganado Nelore, indica que variables como ésta se podrían utilizar como criterio de selección en programas nacionales para mejorar la fertilidad del hato(6). Asimismo, Berry y Evans ${ }^{(7)}$ encontraron en una población de animales cruzados Bos taurus que la variación genética en características reproductivas era suficiente para asegurar un programa exitoso de mejora genética del comportamiento reproductivo.

En México, se han realizado evaluaciones genéticas nacionales para la Asociación Mexicana Simmental-Simbrah, A.C. y la Charolais Charbray Herd Book de México, A.C., desde el 2001 y 2002, respectivamente. Estas evaluaciones se han hecho para características de crecimiento, circunferencia escrotal, talla y permanencia productiva, utilizando procedimientos multirraciales ${ }^{(8)}$, pero no se ha realizado para fertilidad de vaquillas. El objetivo del presente estudio fue desarrollar un prototipo de evaluación genética multirracial para la fertilidad de vaquillas de las poblaciones Simmental-Simbrah y Charolais-Charbray.

\section{MATERIAL Y MÉTODOS}

\section{Población de estudio}

Se utilizó la información de 49,943 hembras registradas en la Asociación Mexicana SimmentalSimbrah A.C., nacidas entre 1984 y 2011 (EvSim), y la de 49,871 hembras registradas en la Charolais Charbray Herd Book de México A.C., nacidas entre 1990 y 2011 (EvChar). Los registros de las asociaciones indicaron que no se realizó cambio de propietario de las hembras incluidas en las presentes evaluaciones genéticas, por lo que se consideró que permanecieron en el mismo grupo contemporáneo desde el nacimiento hasta los 1,281 días de edad (42 meses). Se definieron cuatro épocas en función de la distribución de los nacimientos de las vaquillas: enero-marzo, abril-junio, julio-septiembre y octubrediciembre. El grupo contemporáneo (GC) se integró por hato, año y época de nacimiento de la vaquilla. Los GC con menos de cuatro registros no fueron considerados en los análisis. La edad de la madre de la vaquilla al parto se calculó en días. Los Cuadros 1 y 2 resumen la estructura de las bases de datos utilizadas.

\section{Variable de respuesta}

La característica evaluada fue la fertilidad de vaquillas (FERT); para cada vaquilla se generó una variable en función de su desempeño reproductivo; a las que parieron antes de los 1,281 días de edad se les asignó un uno y en caso contrario un cero.

\section{Modelos estadísticos}

Para determinar si los efectos fijos incluidos en el modelo lineal mixto definitivo explicaban variación significativa, se ajustaron los modelos lineales 
Cuadro 1. Estructura general de los datos y de los pedigríes utilizados para evaluar la fertilidad de vaquillas en poblaciones Simmental-Simbrah y Charolais-Charbray

\begin{tabular}{lcc}
\hline & Simmental-Simbrah & Charolais-Charbray \\
\hline Animales en el pedigrí & 75,926 & 72,486 \\
Animales con registros productivos & 49,943 & 49,871 \\
Sementales en el pedigrí & 4,976 & 5,741 \\
Sementales con progenie con registros productivos & 4,335 & 3,592 \\
Madres en el pedigrí & 37,756 & 41,467 \\
Madres con registros & 33,890 & 30,979 \\
\hline
\end{tabular}

Cuadro 2. Medias, mínimos y máximos de la información utilizada para evaluar la fertilidad de vaquillas en poblaciones Simmental-Simbrah y Charolais-Charbray

\begin{tabular}{lrrr}
\hline & Media & Min & Max \\
\hline Simmental-Simbrah: & & & \\
Crías por semental & 11.52 & 1 & 315 \\
Sementales por año y hato & 3.52 & 1 & 59 \\
Años activos por semental & 2.51 & 1 & 19 \\
Vacas por semental & 10.87 & 1 & 256 \\
Crías por vaca & 1.47 & 1 & 9 \\
Charolais-Charbray: & & & \\
Crías por semental & 13.88 & 1 & 346 \\
Sementales por año y hato & 5.19 & 1 & 31 \\
Años activos por semental & 2.61 & 1 & 16 \\
Vacas por semental & 13.03 & 1 & 336 \\
Crías por vaca & 1.61 & 1 & 8 \\
\hline
\end{tabular}

preliminares $^{(9,10)}$ con el procedimiento GLM del paquete estadístico $\mathrm{SAS}^{(11)}$. De esta manera se decidió incluir la edad de la madre al parto como covariable lineal y cuadrática. El modelo lineal mixto definitivo, empleado en ambas evaluaciones genéticas, quedó estructurado como se muestra a continuación:

$$
\mathrm{y}=\mathrm{Xb}+\mathrm{Za}+\mathrm{e},
$$

Donde, y es el vector de observaciones de FERT; b es el vector de efectos fijos para GC $(4,938$ y 4,528 niveles para EvSim y EvChar, respectivamente), edad de la madre de la vaquilla al parto, en días, como covariable lineal y cuadrática (EM y EM2, respectivamente), porcentaje de genes Simmental o Charolais (PG), heterocigosis (HET) y pérdidas por recombinación (PR) como covariables relacionadas con el genotipo de la vaquilla ${ }^{(12,13)}$; a es un vector aleatorio de efectos genéticos aditivos directos (ANIM); e es un vector aleatorio de efectos residuales; $\mathbf{X}$ y $\mathbf{Z}$ son matrices de incidencia que asocian los registros de FERT con los correspondientes efectos. Se asumió que los efectos aleatorios siguieron una distribución normal multivariada, con medias iguales a cero y con la estructura de (co)varianzas siguiente:

$$
\left(\begin{array}{l}
a \\
e
\end{array} \mid \sigma_{\mathrm{a}}^{2}, \sigma_{\mathrm{e}}^{2}\right) \sim N\left[0,\left(\begin{array}{cc}
A \sigma_{\mathrm{a}}^{2} & 0 \\
0 & \mathrm{I}_{\mathrm{n}} \sigma_{\mathrm{e}}^{2}
\end{array}\right)\right]
$$

Donde, A es la matriz de parentescos entre los animales que conformaron el pedigrí (de tamaño 75,926 x 75,926 para EvSim, y 74,486 x 74,486 para EvChar), $\boldsymbol{\sigma}_{\mathbf{a}}^{2}$ es la varianza genética aditiva directa, $\boldsymbol{\sigma}_{\mathbf{e}}^{2}$ es la varianza residual e $\mathbf{I}_{\mathbf{e}}$ es una matriz identidad de tamaño igual al número de registros $(49,943$ y 49,871 para EvSim y EvChar, 
respectivamente). Además, se asumió que los efectos genético aditivo directo y residual fueron independientes ${ }^{(14,15)}$.

\section{Análisis estadísticos}

Los estimadores de los componentes de varianza, los valores del logaritmo de la verosimilitud ( $\log \mathrm{L})$ y las DEP se obtuvieron ajustando un modelo lineal mixto para una sola característica. En la estimación se asumió que FERT se distribuyó binomialmente. Los análisis se realizaron con el paquete estadístico ASREML, utilizando el algoritmo de información promedio de máxima verosimilitud restringida ${ }^{(16)}$. Se asumió convergencia cuando el logaritmo de la verosimilitud cambió menos de 0.002 y el estimador del parámetro de la varianza menos del $1 \%$.

La fórmula utilizada para el cálculo de la heredabilidad $\left(h^{2}\right)$ fue:

$$
h^{2}=\frac{\sigma_{a}^{2}}{\sigma_{a}^{2}+\sigma_{e}^{2}}
$$

La DEP es el mejor estimador del mérito genético de un individuo, como esperanza del verdadero valor genético y debe tener un valor de confiabilidad asociado, que tiene una relación directa con la cantidad de información disponible para cada individuo. La confiabilidad se calculó con la siguiente fórmula ${ }^{(17):}$

$$
1-\sqrt{\frac{\text { Varianza del error de predicción }}{\text { Varianza genética aditiva }}}
$$

Con la finalidad de evaluar el cambio de FERT a través del tiempo en las poblaciones estudiadas, se obtuvieron los promedios de las DEP por año de nacimiento de las vaquillas entre 1984 y 2011 . Las tendencias genéticas se estimaron mediante la regresión lineal simple de estas medias en los años correspondientes, y la significancia de los coeficientes de regresión obtenidos se probó con el estadístico $t^{(18)}$, usando el procedimiento REG de SAS ${ }^{(11)}$.

Se compararon los promedios de las DEP y de las confiabilidades, de la totalidad de sementales y de subconjuntos con el $20,10,5$ y $1 \%$ superior de los sementales.

\section{RESULTADOS Y DISCUSIÓN}

\section{Componentes de varianza y heredabilidad}

Los estimadores de los componentes de varianza y heredabilidad para la fertilidad de vaquillas se presentan en el Cuadro 3 . Los estimadores de heredabilidad para ambas evaluaciones fueron similares, $0.07 \pm 0.008$ (EvSim) y $0.06 \pm 0.008$ (EvChar). Estos valores están dentro del intervalo de estimadores reportados por otros investigadores que utilizaron modelos lineales. En una población multirracial, se estimó que la heredabilidad de la tasa de parto fue $0.06 \pm 0.02^{(15)}$. En un programa de selección a largo plazo para características de crecimiento, con líneas Hereford, también se obtuvo una heredabilidad de $0.06 \pm 0.06$ para tasa de preñez ${ }^{(19)}$. Resultados similares se obtuvieron para Hereford y cruzas de Shorthorn y Hereford con Cebú (0.079 y 0.081, respectivamente); sin embargo, heredabilidades para tasa de parto muy próximas a cero se

Cuadro 3. Estimadores de componentes de varianza y heredabilidad de la fertilidad de vaquillas

\begin{tabular}{l|c|c}
\hline & Simmental-Simbrah & Charolais-Charbray \\
Varianza aditiva & $0.0103(0.0012)$ & $0.0079(0.0011)$ \\
Varianza del error & $0.1326(0.0013)$ & $0.1348(0.0013)$ \\
Varianza fenotípica & $0.1429(0.0009)$ & $0.1428(0.0009)$ \\
Heredabilidad & $0.0721(0.0082)$ & $0.0594(0.0077)$ \\
$\log L$ & $6,223.74$ & $6,644.90$ \\
\hline
\end{tabular}

Errores estándar entre paréntesis. 
calcularon para Angus, $0.015^{(20)}$. En un estudio realizado con Angus, la heredabilidad del porcentaje de preñez de vaquillas de dos años fue de $0.08 \pm$ 0.06 , similar a la encontrada en este trabajo(21). En contraste, en la raza Simmental, se estimaron heredabilidades para fertilidad de vaquillas menores a las del presente trabajo, $0.02 \pm 0.003$ cuando se usó monta natural y $0.016 \pm 0.0025$ cuando se usó inseminación artificial(5).

Un estimador ligeramente mayor $(0.11 \pm 0.03)$ que el obtenido en el presente estudio, se encontró en una población Nelore en clima tropical, sujeta a selección para peso al año; los autores concluyeron que el avance genético de la fertilidad no se vio comprometido por la mejora en la característica de

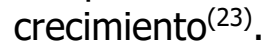

En contraste, Silva et $a^{(22)}$ encontraron heredabilidades para preñez de 0.12 en vaquillas que parieron antes de los 46 meses y de 0.52 para las que parieron antes de los 31 meses. Diferencias en heredabilidad de la fertilidad se esperan entre poblaciones Bos taurus y Nelore, dado que estas últimas han sido sometidas a menor intensidad de selección para mejorar la fertilidad(24). Por otro lado, estimadores de heredabilidad obtenidos con modelos umbrales son mayores, de 0.21 a $0.53^{(24-28)}$.

Los estimadores de heredabilidad del presente trabajo indican que la expresión de FERT dependió en gran proporción de factores ambientales. Sin embargo, aunque las heredabilidades estimadas en este estudio son bajas, el intervalo observado de las DEP (Cuadro 4) muestra que puede haber respuesta genética de FERT a la selección. Amplitudes ligeramente mayores entre las DEP de sementales se observaron en una población multirracial $(-11.3$ a $12.2)^{(15)}$ y en una población Angus (-11.94 a

Cuadro 4. Diferencias esperadas en la progenie (DEP) y confiabilidades estimadas para grupos de sementales superiores para las evaluaciones Simmental-Simbrah y Charolais-Charbray

\begin{tabular}{|c|c|c|c|c|c|c|}
\hline & $\mathrm{n}$ & $\operatorname{DEP}(\%)$ & DE & Min & Max & Confiabilidad \\
\hline \multicolumn{7}{|c|}{ Sementales Simmental } \\
\hline 1\% superior & 11 & 6.13 & 1.22 & 5.08 & 8.72 & 0.14 \\
\hline $5 \%$ superior & 53 & 3.76 & 1.48 & 2.2 & 8.72 & 0.10 \\
\hline $10 \%$ superior & 105 & 2.81 & 1.43 & 1.6 & 8.72 & 0.08 \\
\hline $20 \%$ superior & 209 & 2.01 & 1.29 & 0.89 & 8.72 & 0.06 \\
\hline $100 \%$ & 1,052 & -0.18 & 1.62 & -5.79 & 8.72 & 0.05 \\
\hline \multicolumn{7}{|c|}{ Sementales Simbrah } \\
\hline $1 \%$ superior & 9 & 6.94 & 1.05 & 5.64 & 8.84 & 0.18 \\
\hline $5 \%$ superior & 46 & 4.41 & 1.48 & 2.88 & 8.84 & 0.13 \\
\hline $10 \%$ superior & 92 & 3.34 & 1.51 & 1.84 & 8.84 & 0.10 \\
\hline $20 \%$ superior & 185 & 2.37 & 1.44 & 1.08 & 8.84 & 0.09 \\
\hline $100 \%$ & 924 & -0.06 & 1.78 & -9.56 & 8.84 & 0.06 \\
\hline \multicolumn{7}{|c|}{ Sementales Charolais } \\
\hline $1 \%$ superior & 27 & 5.07 & 0.86 & 4.27 & 8.22 & 0.13 \\
\hline $5 \%$ superior & 136 & 3.38 & 1.04 & 2.26 & 8.22 & 0.10 \\
\hline $10 \%$ superior & 272 & 2.59 & 1.09 & 1.41 & 8.22 & 0.09 \\
\hline $20 \%$ superior & 545 & 1.80 & 1.11 & 0.72 & 8.22 & 0.07 \\
\hline $100 \%$ & 2,717 & -0.07 & 1.39 & -7.94 & 8.22 & 0.05 \\
\hline \multicolumn{7}{|c|}{ Sementales Charbray } \\
\hline $1 \%$ superior & 2 & 5.59 & 0.76 & 5.06 & 6.13 & 0.17 \\
\hline $5 \%$ superior & 5 & 4.51 & 1.07 & 3.59 & 6.13 & 0.13 \\
\hline $10 \%$ superior & 11 & 3.51 & 1.20 & 2.10 & 6.13 & 0.10 \\
\hline $20 \%$ superior & 21 & 2.59 & 1.32 & 1.17 & 6.13 & 0.09 \\
\hline $100 \%$ & 104 & 0.04 & 1.84 & -7.29 & 6.14 & 0.07 \\
\hline
\end{tabular}

n= número de sementales; $\mathrm{DE}=$ desviación estándar; $\mathrm{MIN}=$ valor mínimo de las $\mathrm{DEP} ; \mathrm{MAX}=$ valor máximo de las DEP. 
10.01)(26); los autores opinaron que estas variaciones son suficientes para seleccionar con el objetivo de aumentar la tasa reproductiva.

\section{Grupos contemporáneos}

Los efectos no genéticos han sido incluidos en los modelos de predicción para remover sesgos en las evaluaciones genéticas. El grupo contemporáneo se incluye para minimizar la variación que ocasionan los cambios en las condiciones ambientales del hato, que se dan por las asociaciones entre los grupos

Figura 1. Distribución de los grupos contemporáneos en función del número de vaquillas para la evaluación Simmental-Simbrah (A) y Charolais-Charbray (B)
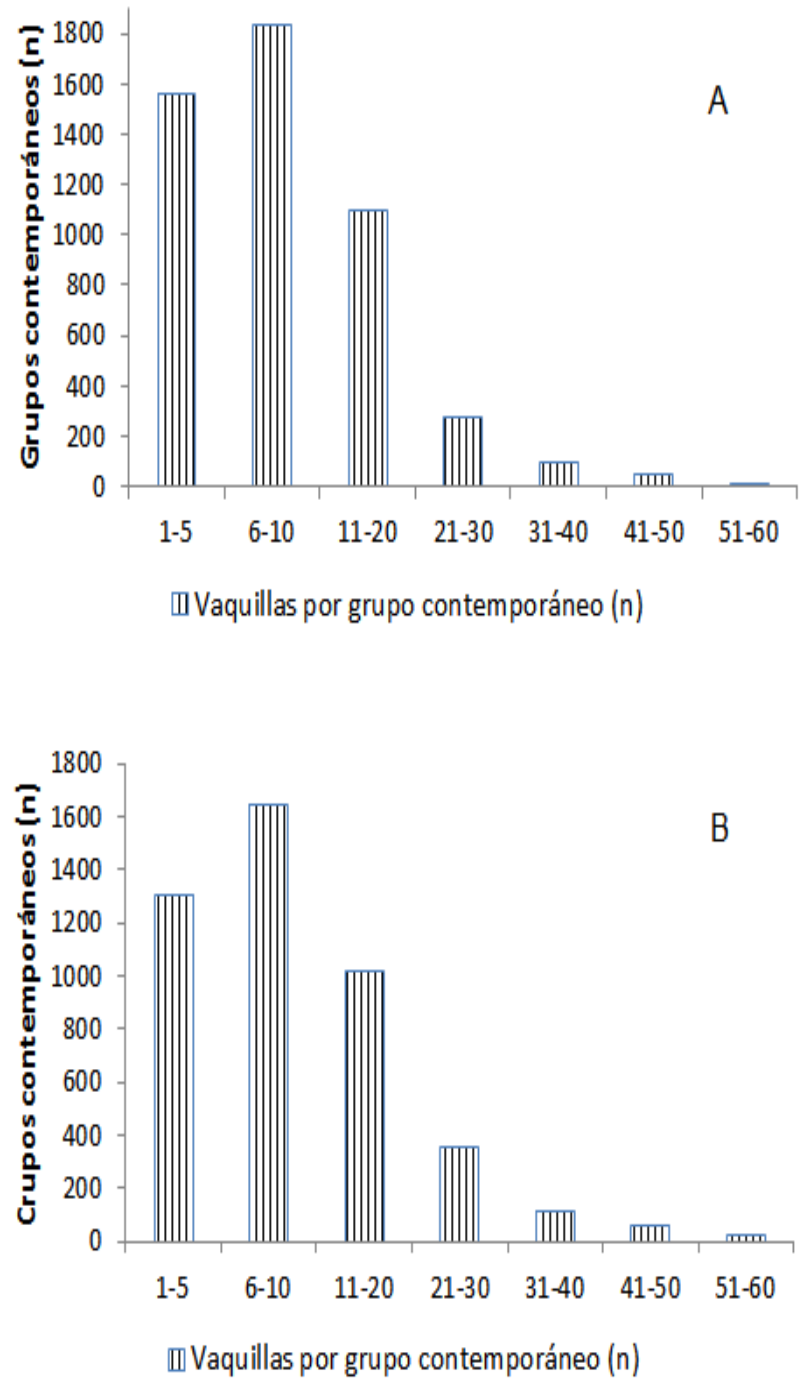

contemporáneos y el nivel genético de los animales $^{(29)}$. La varianza del error de predicción es afectada por el tamaño de los grupos contemporáneos, así como por el tamaño de las familias utilizadas en la evaluación. La disminución del tamaño de los grupos contemporáneos aumenta el número de grupos contemporáneos que deben ser estimados y los grados de libertad perdidos en el proceso. En la Figura 1 se presenta la distribución del número de hembras en grupos contemporáneos de EvSim y EvChar, respectivamente. Las medianas del número de hembras por grupo contemporáneo de EvSim y EvChar fueron similares, con 10, en un rango intercuartil de 5 a 20. En el presente trabajo se encontró, en ambas poblaciones, que la confiabilidad de los valores genéticos disminuyó a medida que decreció el tamaño del grupo contemporáneo. Los valores de confiabilidad más bajos de los sementales estuvieron por debajo de 0.12 y 0.08 para EvSim y EvChar, respectivamente, cuando el tamaño del grupo contemporáneo fue menor de 10. Ugarte et $a^{(29)}$ encontraron que la cantidad efectiva de información para la estimación de valores genéticos disminuyó con el tamaño del grupo contemporáneo, lo que aumentó el error de predicción, aun cuando el número de hijas por semental fue constante y los mayores valores de estos fueron de grupos contemporáneos menores de 9.

\section{Grupos familiares}

El número de sementales utilizados en el presente estudio fue 4,335 y 3,592 para EvSim y EvChar, respectivamente. La distribución del tamaño de las familias paternas de las dos evaluaciones se presenta en la Figura 2 . La relación entre el tamaño de los grupos contemporáneos y el de las familias de medias hermanas utilizadas en la evaluación genética determina los niveles de confiabilidad y el sesgo de los valores genéticos obtenidos. El tamaño óptimo de las familias de medios hermanos para la estimación de la heredabilidad está determinado por la relación $4 / \mathrm{h}^{2(30,31)}$. Bajos estimadores de heredabilidad se obtuvieron en ambas evaluaciones, 0.07 y 0.06 para EvSim y EvChar, respectivamente. La relación entre el número de hijas y la confiabilidad 
Figura 2. Distribución del tamaño de las familias paternas de las vaquillas en la evaluación Simmental-Simbrah (A) y Charolais-Charbray (B)
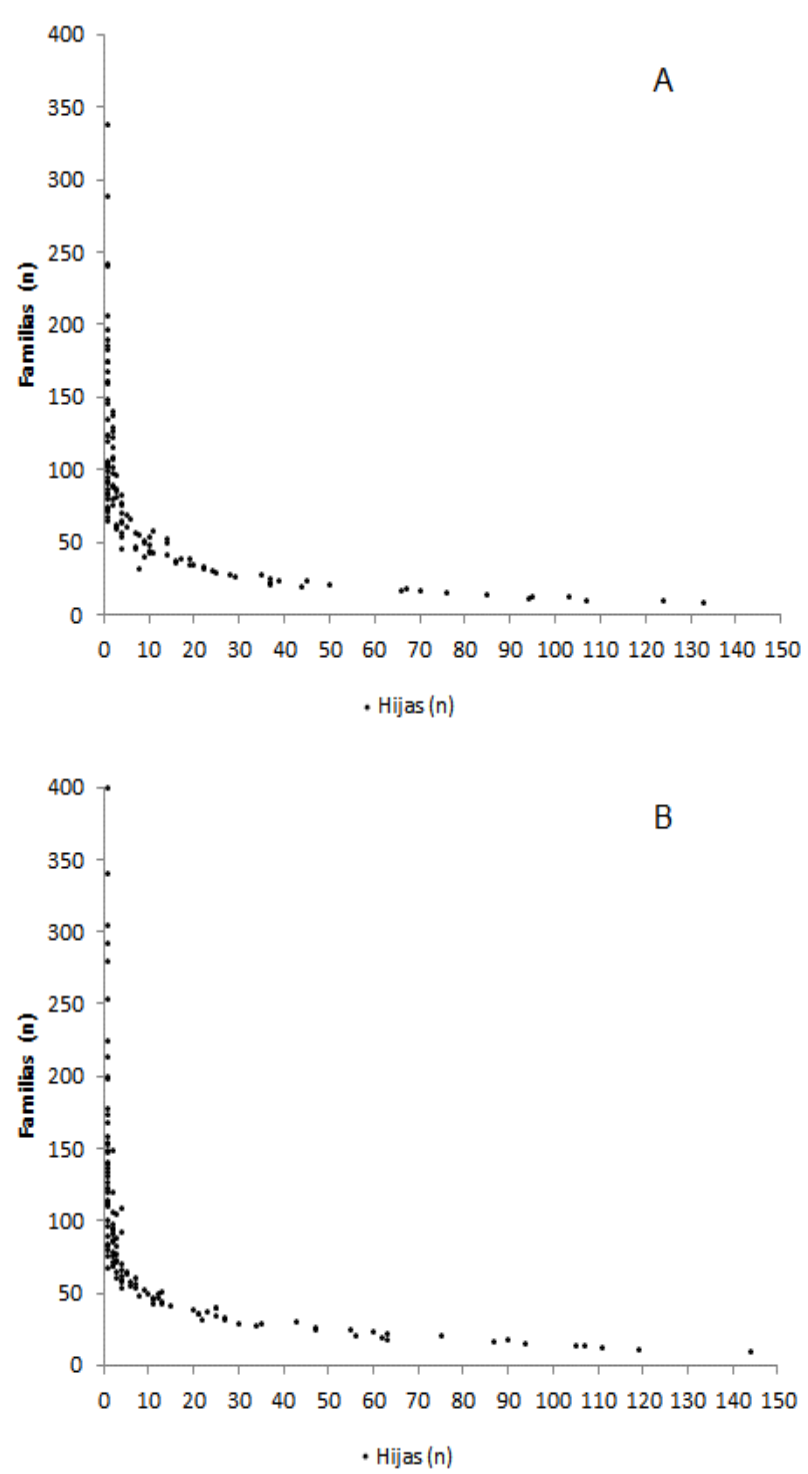

de las DEP de los sementales se presenta en la Figura 3. En ésta se puede observar que para alcanzar niveles de confiabilidad superiores a 0.3 se requiere que un semental tenga más de 100 hijas. En un estudio de reproducción sostenida exitosa, se encontró media y mediana de tamaño de familia de 7 y 11 , respectivamente; también estimaron que para alcanzar una confiabilidad de 0.5 un semental necesitaría 90 hijas, considerando la heredabilidad
Figura 3. Relación entre la confiabilidad y el tamaño de familia paterna para la evaluación Simmental- Simbrah (A) y Charolais-Charbray (B)
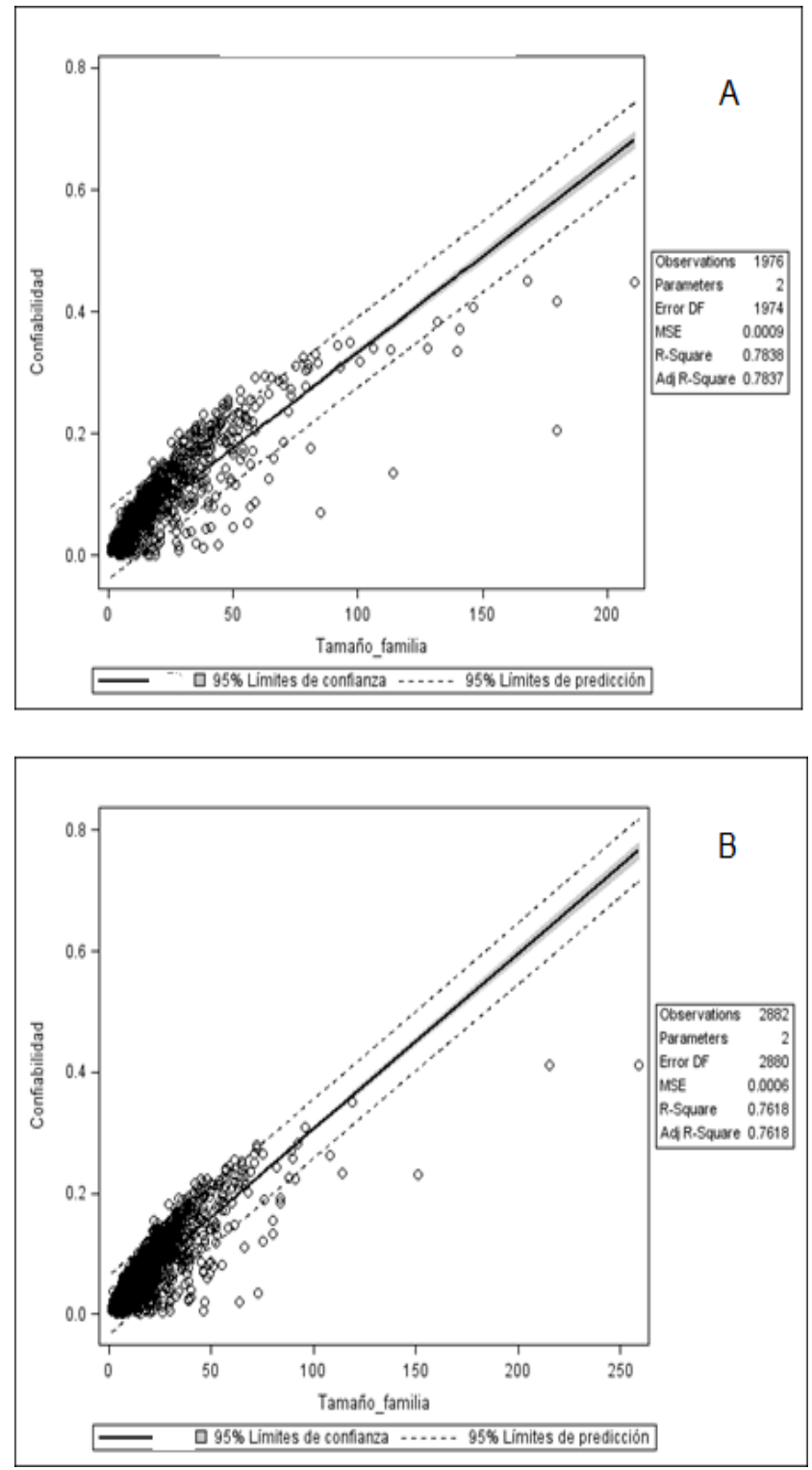

de 0.05; por lo que los investigadores determinaron que para mejorar la confiabilidad se requiere que el tamaño de las familias aumente sustancialmente ${ }^{(31)}$. Como alternativa para evitar el descarte de información proveniente de GC pequeños, en especial de hijas de toros jóvenes, investigadores proponen la utilización combinada GC fijos y 
aleatorios selectivos, en donde han encontrado que su utilización interfiere poco en el ordenamiento de los valores genéticos, la estimación de los parámetros genéticos y las tendencias genéticas ${ }^{(32)}$.

Figura 4. Tendencia genética de la fertilidad de vaquillas para la evaluación Simmental-Simbrah (A) y CharolaisCharbray (B)
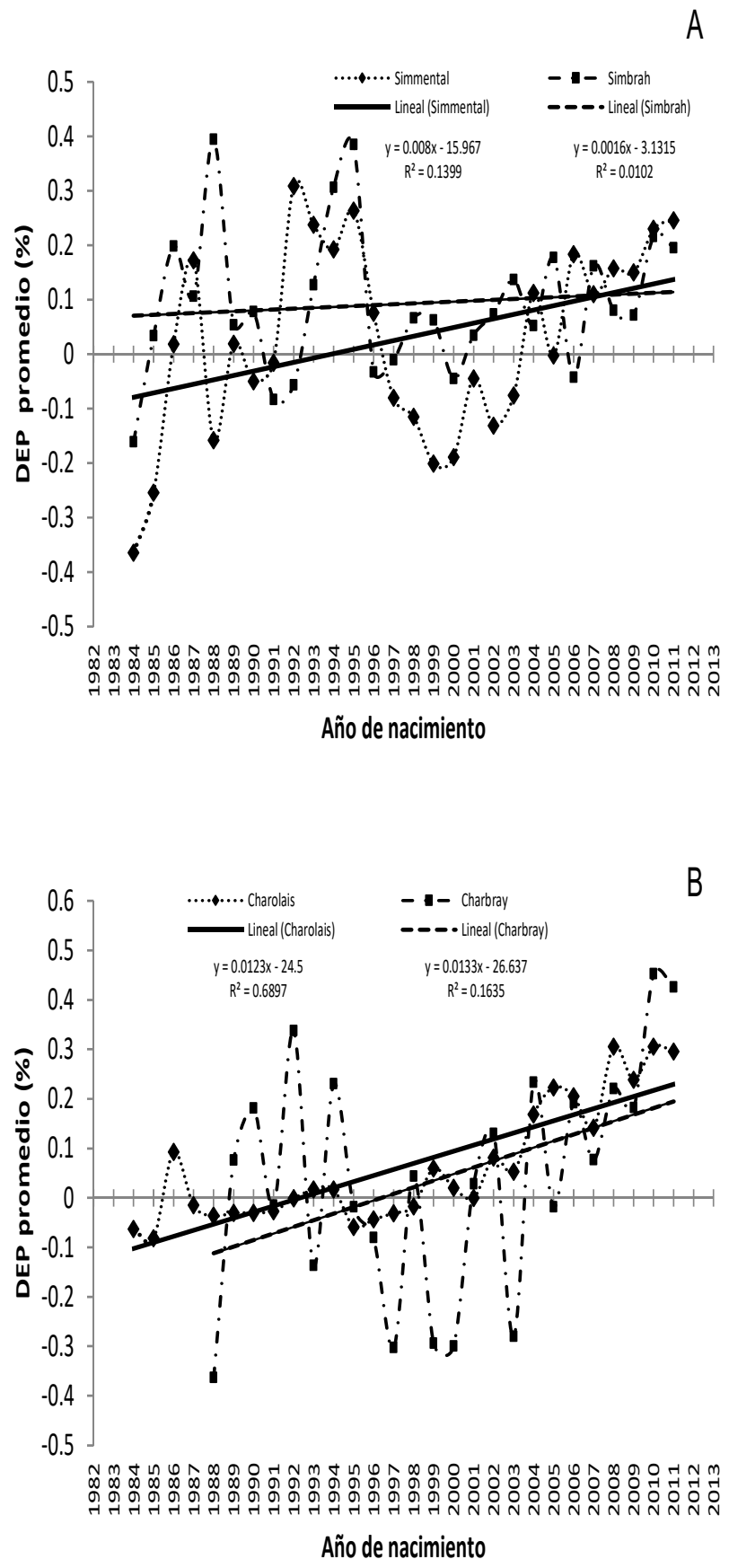

\section{Tendencias genéticas}

La tendencia genética anual en la fertilidad de vaquillas es el cambio en el mérito genético aditivo en la población para esta característica en el tiempo. En la Figura 4 se presentan las tendencias de las DEP para FERT, a través de los años, de las razas Simmental, Simbrah, Charolais y Charbray. Tanto para EvSim como para EvChar las DEP fueron ajustadas por el promedio de las DEP del año 2001; este año se consideró como la base genética en ambas poblaciones. El cambio genético anual para Charolais, Simmental y Simbrah, entre los años de 1984 y 2011, se estimó en $0.012 \pm 0.001(P<0.001)$, $0.008 \pm 0.004(P<0.05)$ y $0.002 \pm 0.003$ puntos porcentuales $(P>0.05)$, y las medias generales ajustadas de las DEP para este período fueron 0.063 $\pm 0.069,0.028 \pm 0.166$ y $0.09 \pm 0.134$, respectivamente. En 1984, la DEP promedio de FERT fue $-0.063,-0.36$ y -0.16 , para las poblaciones Charolais, Simmental y Simbrah, respectivamente. A partir de este año, el promedio de las DEP incrementó de manera uniforme hasta llegar, en el 2011 a 0.29 en Charolais, 0.25 en Simmental y 0.19 en Simbrah. También se observa la tendencia genética de la población Charbray con un patrón errático, que coincide con los años de menor cantidad de información disponible de esta raza. El cambio genético anual observado en Charbray entre 1988 y 2011 fue el mayor de entre las cuatro razas $(0.1334 \pm 0.006(P<0.05)$; el promedio de las DEP fue $0.041 \pm 0.219$. A partir del 2001, la tendencia de FERT para la población Charbray siguió una pauta similar a las de las demás razas evaluadas. Es importante resaltar que las tendencias genéticas estimadas en ambas poblaciones muestran que existió una respuesta genética favorable de la fertilidad de las vaquillas de las cuatro razas evaluadas, durante el período 2007-2011.

En general, se observó un ligero cambio genético en las poblaciones en estudio, aun cuando se ha aplicado mayor presión de selección sobre las características de crecimiento, como el peso al destete, donde las DEP se han incrementado en el tiempo ${ }^{(33)}$. Resultados similares se encontraron en un estudio de selección para peso al año en Nelore, donde las medias anuales de los valores genéticos para tasa de parto a primer servicio se mantuvieron 
prácticamente sin cambio durante 15 años(23); estos autores concluyeron que las ganancias significativas en peso corporal del año en adelante, como resultado de su proceso de selección, no interfirieron en el comportamiento reproductivo de las vacas, en específico de los días al parto y la tasa de parto. Al comparar los resultados obtenidos en el presente trabajo con los de un estudio de tendencias genéticas para tasa de no retorno de vaquillas Noruegas, se encontró que el cambio genético fue positivo $(0.16 \%)$ y solo fue similar a la tendencia observada en Charbray ${ }^{(34)}$.

\section{Ordenamiento de sementales}

En el Cuadro 4 se presentan los estadísticos de los grupos de sementales ordenados en rangos por su mayor nivel de DEP para EvSim y EvChar. El intervalo de fluctuación de las DEP fue similar para Simmental, Simbrah y Charolais, donde el valor máximo de las DEP estuvo alrededor de los 8 puntos porcentuales; en cambio el intervalo en Charbray fue menor. En la Figura 3 se observa que las confiabilidades más altas estuvieron por arriba de 0.40 , en los grupos familiares con más de 100 individuos. Aun cuando la heredabilidad estimada de FERT para las poblaciones estudiadas fue baja, habría que tomar en cuenta la amplitud existente entre las DEP de los sementales. Para ponerlo en contexto, consideremos que un semental que tiene una DEP de $8 \%$ tiene la posibilidad de producir hijas con un $7 \%$ más de probabilidad de parir a los 42 meses de edad que las hijas de otro semental con una DEP de $1 \%$.

En un estudio con Angus $^{(35)}$, las DEP para porcentaje de preñez fluctuaron entre -0.02 y 0.01 , intervalo que fue menor al observado en el presente trabajo. Estos autores concluyeron que, aunque la heredabilidad fue baja (0.12), con la variabilidad de los valores de cría obtenidos se podría lograr progreso genético mediante selección. Por otro lado, intervalos de DEP más amplios (de -11.3 a $12.2 \%$ ) se estimaron en una población multirracial Bos taurus, para porcentaje de parto ${ }^{(15)}$. Estos autores también determinaron que el intervalo de las DEP no fue afectado por el tipo de modelo estadístico que se utilizó, lineal, umbral o logístico. Con vaquillas Nelore y un modelo umbral, otros investigadores encontraron que el intervalo de las DEP y los valores de éstas fueron mayores que los que se obtuvieron en el presente trabajo (-24.5 a 24.55); atribuyendo estos resultados tanto al método de estimación utilizado, como a la mayor variabilidad genética de la fertilidad de las vaquillas en la población estudiada(36).

\section{CONCLUSIONES E IMPLICACIONES}

Las heredabilidades estimadas indican que puede haber respuesta genética a la selección de FERT en las poblaciones evaluadas. Las tendencias genéticas estimadas muestran un cambio genético favorable de la fertilidad de vaquillas Simmental, Simbrah, Charolais y Charbray durante el período 2007-2011. Los rangos de los valores de las DEP permiten identificar sementales sobresalientes para la fertilidad de sus hijas en las cuatro razas estudiadas.

\section{LITERATURA CITADA}

1. Dickerson G. Efficiency of animal production - Molding the biological components. J Anim Sci 1970;30:849-859.

2. Van Eenennaam AL. Considerations related to breed or biological type. Vet Clin Food Anim 2013;29(3):493-516.

3. Cammack KM, Thomas MG, Enns RM. Review: reproductive traits and their heritabilities in beef cattle. Prof Anim Sci 2009;25:517528.

4. Johnston DJ. Genetic improvement of reproduction in beef cattle. Proc $10^{\mathrm{TH}}$ World congress of genetics applied to livestock production. Vancouver, Canada. 2014.

5. Jamrozik J, McGrath S, Kemp RA, Miller SP. Genetic analysis of female fertility traits in Canadian Simmentals. Livest Sci 2012;150:302-309.

6. Shiotsuki L, Silva JAIIV, Tonhati, Albuquerque LG. Genetic association of sexual precocity with growth traits and visual scores of conformation, finishing, and muscling in Nelore cattle. J Anim Sci 2009;87:1591-1597.

7. Berry DP, Evans RD. Genetics of reproductive performance in seasonal calving beef cows and its association with performance traits. J Anim Sci 2014;92:1412-1422.

8. Román PSI, Ríos UA, Montaño BM, García RA, Vega MVE, Sifuentes RAM, et al. Mejoramiento genético de los bovinos en el trópico. En: González PE, et al editores. Estado del arte sobre investigación e innovación tecnológica en ganadería bovino tropical. $1^{\mathrm{a}}$ ed. REDGATRO-CONACYT. 2015:99-152.

9. Ghorbani S, Tahmoorespur M, Maghsoudib A, Abdollahi-Arpanahi R. Estimates of (co)variance components for production and 
reproduction traits with different models in Fars native fowls. Livest Sci 2013;151:115-123.

10. Orenge JSK, Ilatsia ED, Kosgey IS, Kahi AK. Genetic and phenotypic parameters and annual trends for growth and fertility traits of Charolais and Hereford beef cattle breeds in Kenya. Trop Anim Health Prod 2009;41:767-774.

11. SAS. SAS/STAT software, Version 9.3 for Windows. Copyright (c) 2002-2010 SAS Institute Inc. SAS and all other SAS Institute Inc. product or service names are registered trademarks or trademarks of SAS Institute Inc., Cary, NC, USA. 2010.

12. Dickerson GE. Experimental approaches in utilizing breed resources. Anim Breed Abstr 1969;37:191-202.

13. Dickerson G. Inbreeding and heterosis in animals. In: Proc Anim Breed Gen Symp in honor of Dr. Jay L. Lush. ASAS,ADSA and PSA, Champaing, IL. 1973.

14. Williams, R. Comparing logit and probit coefficients between models and across groups 2011. https://www3.nd.edu/ rwilliam/ stats/Oglm.pdf. Accessed 21 Jul, 2016.

15. Guerra JLL, Franke DE, Blouin DC. Genetic parameters for calving rate and calf survival from linear, threshold, and logistic models in a multibred beef cattle population. J Anim Sci 2006;84:3197-3203.

16. Meyer K. PX $\times$ AI: Algorithmics for better convergence in restricted maximum likelihood estimation. Proc World Congr Genet Appl Livest Prod 2006; Comm. No. 24-00.

17. Bertrand K, Cundiff L, Golden B, Kachman SD, Quaas R, Van Vleck D, Williams RE. National cattle evaluation. In: Cundiff LV, Van Vleck LD, Hohenboken WD. Guidelines for uniform beef improvement programs. 9th ed. Beef Improvement Federation. Raleigh, USA: North Carolina State University; 2010;65-82.

18. Bernardes PA, Grossi DA, Savegnago RP, Buzanskas ME, Urbinati I, Bezerra LAF, et al. Estimates of genetic parameters and genetic trends for reproductive traits and weaning weight in Tabapuã cattle. J Anim Sci 2015;93:5175-5185.

19. Toelle VD, Robinson OW. Estimates of genetic correlations between testicular measurements and female reproductive traits in cattle. J Anim Sci 1985;60(1):89-100.

20. Meyer K. Hammond K, Parnell PF, Mackinnon MJ, Sivarajasingam S. Estimates of heritability and repeatability for reproductive traits in Australian beef cattle. Livest Prod Sci 1990;25:15-30.

21. Morris CA, Wilson JA, Bennett GL, Cullen NG, Hickey SM, Hunter JC. Genetic parameters for growth, puberty, and beef cow reproductive traits in a puberty selection experiment. N Z J Agr Res 2000;43:8391.

22. Silva JAIIV, Dias LT, Albuquerque LG. Estudo genético da precocidade sexual de novilhas em um rebanho Nelore. R Bras Zootec 2005;34:1568-1572.
23. Mercadante MEZ, Packer UI, Razook AG, Cyrilo JNSG, Figueiredo LA. Direct and correlated response to selection for yearling weight on reproductive performance of Nelore cows. J Anim Sci 2003;81:376-384.

24. Boligon AA, Albuquerque LG. Genetic parameters and relationships of heifer pregnancy and age at first calving with weight gain, yearling and mature weight in Nelore cattle. Livest Sci 2011;141:12-16.

25. Buddenberg BJ, Brown CJ, Johnson ZB, Dunn JE, Peterson HP. Heritability estimates of pregnancy rate in beef cows under natural mating. J Anim Sci 1989;67:2589-2594.

26. Doyle SP, Golden BL, Green RD, Brinks JS. Additive genetic parameter estimates for heifer pregnancy and subsequent reproduction in Angus females. J Anim Sci 2000;78:2091-2098.

27. Snelling WM, MacNeil MD, Golden BL. Application of continuous and binary trait methods to reproductive measures of Hereford cattle. J Anim Sci 1996;74(Suppl 1):115.

28. Van Melis MH, Eler JP, Rosa GJM, Ferraz JBS, Figueiredo LGG, Mattos EC, Oliveira HN. Additive genetic relationships between scrotal circumference, heifer pregnancy, and stayability in Nellore cattle. J Anim Sci 2010;88:3809-3813.

29. Ugarte $E$, Alenda R, Carabano MJ. Fixed or random contemporary groups in genetic evaluations. J Dairy Sci 1992;75:269-278.

30. Robertson A. Experimental design in the evaluation of genetic parameters. Biometrics 1959;15:219-226.

31. MacNeil, MD, Vukasinovic N. A prototype national cattle evaluation for sustained reproductive success in Hereford cattle. J Anim Sci 2011;89:1712-1718.

32. Santana ML Jr, Eler JP, Ferraz JBS. Alternative contemporary group structure to maximize the use of field records: Application to growth traits of composite beef cattle. Livest Sci 2013;157:20-27.

33. Asociación Mexicana Simmental-Simbrah. Sumario de sementales 2015. http://www.simmentalsimbrah.com.mx/sumario_sementales/ sumario_sementales.pdf. Consultado 8 Ago,2016.

34. Andersen-Ranberg IM, Klemetsdal G, Heringstad B. Short communication: validation of two animal models for estimation of genetic trends for female fertility in Norwegian dairy cattle. J Dairy Sci 2003;86:4090-4092.

35. Bormann JM, Totir LR, Kachman SD, Fernando RL, Wilson DE. Pregnancy rate and first-service conception rate in Angus heifers. ] Anim Sci 2006;84:2022-2025.

36. Eler JP, Silva JA, Ferraz JB, Dias F, Oliveira HN, Evans JL, Golden BL. Genetic evaluation of the probability of pregnancy at 14 months for Nellore heifers. J Anim Sci 2002;80:951-954. 